\title{
Identification of Candida Species Using MP65 Gene and Evaluation of the Candida albicans MP65 Gene Expression in BALB/C Mice
}

\author{
Farahnaz Bineshian ${ }^{1, *}$; Mohammad Hossien Yadegari ${ }^{2}$; Zohre Sharifi ${ }^{3}$; Mohammadreza \\ Akbari Eidgahi ${ }^{4}$; Reza Nasr ${ }^{4}$ \\ ${ }^{1}$ Department of Mycology, Faculty of Medicine, Semnan University of Medical Sciences, Semnan, IR Iran \\ ${ }^{2}$ Department of Mycology, Faculty of Medical Sciences, Tarbiat Modares University, Tehran, IR Iran \\ ${ }^{3}$ Blood Transfusion Research Center, High Institute for Research and Education in Transfusion Medicine, Tehran, IR Iran \\ ${ }^{4}$ Department of Biotechnology, Faculty of Medicine, Semnan University of Medical Sciences, Semnan, IR Iran \\ *Corresponding author: Farahnaz Bineshian, Department of Mycology, Faculty of Medicine, Semnan University of Medical Sciences, Semnan, IR Iran. Tel: + 98-2313354170, Fax: \\ + 98-2313354161, E-mail: fzbineshian@yahoo.com
}

Received: April 7, 2014; Revised: October 22, 2014; Accepted: November 4, 2014

\begin{abstract}
Background:Systemic candidiasis is a major public health concern. In particular, in immunocompromised people, such as patients with neutropenia, patients with Acquired Immune Deficiency Syndrome (AIDS) and cancer who are undergoing antiballistic chemotherapy or bone marrow transplants, and people with diabetes. Since the clinical signs and symptoms are nonspecific, early diagnosis is often difficult. The 65-kDa mannoprotein (MP65) gene of Candida albicans is appropriate for detection and identification of systemic candidiasis. This gene encodes a putative b-glucanase mannoprotein of $65 \mathrm{kDa}$, which plays a major role in the host-fungus relationship, morphogenesis and pathogenicity.

Objectives:The current study aimed to identify different species of Candida(C.albicans, C. glabrata and C. parapsilosis) using the Polymerase Chain Reaction (PCR) technique and also to evaluate C. albicans MP65 gene expression in BALB/C mice.

Materials and Methods: All yeast isolates were identified on cornmeal agar supplemented with tween-80, germ tube formation in serum, and assimilation of carbon sources in the API 20 C AUX yeast identification system. Polymerase Chain Reaction was performed on all samples using species-specific primers for the MP65 65 kDa gene. After RNA extraction, cDNA synthesis was performed by the Maxime RT Pre Mix kit. Candida albicans MP65 gene expression was evaluated by quantitative Real-Time (q Real-Time) and Real-Time (RT) PCR techniques. The 2- $\Delta \Delta C$ T method was used to analyze relative changes in gene expression of MP65. For statistical analysis, nonparametric Wilcoxon test was applied using the SPSS version 16 software.

Results: Using biochemical methods, one hundred, six and one isolates of clinical samples were determined as C. albicans, C. glabrata and C. parapsilosis, respectively. Species-specific primers for PCR experiments were applied to clinical specimens, and in all cases a single expected band for C. albicans, C. glabrata and C. parapsilosis was obtained (475, 361 and 124 base pairs, respectively). All species isolated by culture methods (100\% positivity) were evaluated with PCR using species-specific primers to identify Candida species. Relative expression of Mp65 genes increased significantly after C. albicans injection into the mice $(\mathrm{P}<0.05)$.

Conclusions: The results of the current study showed that the PCR method is reproducible for rapid identification of Candida species with specific primers. Mp65 gene expression of C. albicans after injection into the mice was 2.3 folds higher than before injection, with this difference being significant. These results indicated that increase of Mp65 gene expression might be an early stage of infection; however definitive conclusions require further studies.
\end{abstract}

Keywords: Candida; Pathology; Molecular; Genes

\section{Background}

The incidence of disease caused by Candida spp. has increased markedly as a result of an increased number of Acquired Immune Deficiency Syndrome (AIDS) patients, organ transplantations, and other immunocompromised patients and neoplastic diseases involving broadspectrum antibiotics, and immunosuppressive drugs (1, 2). Candidemia has been estimated as the fourth most common nosocomial infection with an attributed mortality of about 50\% (3). Early initiation of antifungal therapy is crucial in reducing the mortality of disseminated candidiasis. Early diagnosis is often difficult since most clinical signs and symptoms are non-specific and cultures are often negative or positive results are obtained too late for the initiation of effective antifungal therapy. Blood cultures were reported to be negative in $56 \%$ of autopsy-proven disseminated candidiasis. Consequently, effective treatment is often delayed (4).

Candida albicans is the most common pathogenic species. There has been an increase in the frequency of C. glabrata, and C. krusei and also to a lesser extent, $C$. parapsilosis and C. tropicalis. In addition, non-albicans Candida species resistant to many antifungal drugs have

Copyright (C) 2015, Ahvaz Jundishapur University of Medical Sciences. This is an open-access article distributed under the terms of the Creative Commons Attribution-NonCommercial 4.0 International License (http://creativecommons.org/licenses/by-nc/4.0/) which permits copy and redistribute the material just in noncommercial usages, provided the original work is properly cited. 
Bineshian F et al.

been identified. Thus, accurate identification of Candida species is crucial to determine appropriate antifungal therapy (5). Candida albicans is an opportunistic dimorphic pathogen (6). Its ability to transform from yeast to hyphae is considered as a virulence factor (7). A 65-kDa mannoprotein (MP65) is present in both structural and secretory mannoprotein materials (8). Hyphae formation from yeast cells is a virulence trait that enables the human opportunistic pathogen C. albicans to invade host tissues (9). Furthermore, Mp65p is a putative b-glucanase adhesin with one $\mathrm{N}$ - and multiple potential O-glycosylation sites as a member of the GH17 glycosyl-hydrolase family, which is a major target of immune response.

The MP65 gene of C. albicans plays a major role in the host-fungus relationship, morphogenesis and pathogenicity $(1,3,10-14)$. There is currently an increasing interest in the development of new technological tools for the diagnosis of invasive candidiasis. A shift has been observed in the spectrum of Candida species, with an increase of non-albicans Candida species in clinical isolates. Increasing incidence of candidemia caused by C. parapsilosis and C. glabrata (15), has been described by previous reports. Clinical microbiology laboratories are challenged for rapid and accurate pathogenic Candida detection and identification. Although various laboratory tests have been developed to detect Candida-specific antibodies, antigens, or metabolites, they are time-consuming and lack specificity and/or sensitivity (16).

\section{Objectives}

In the recent years, numerous DNA-based methods have been developed to diagnose Candida infections. Identification of different species of Candida is difficult. The current study describes the development of Polymerase Chain Reaction (PCR) methods for detection of Candida species (C. albicans, C. glabrata and C. parapsilosis) in clinical isolates using primers directed to the MP65 gene. Another objective of the current study was to evaluate the expression of MP65 gene of C. albicans in BALB/C mice.

\section{Materials and Methods}

\subsection{Candida Strains}

In the current study the following reference strains, Candida albicans (ATCC10231), Canddia glabrata (ATCC66032) and Candida parapsilosis (ATCC90018), were used. One hundred and seven clinical isolates were provided by the Tehran University of Medical Sciences, 66 isolates from patients with Human Immunodeficiency Virus (HIV) infected by oral candidiasis, 40 isolates from women with vulvovaginal candidiasis, and one isolate from the Department of Mycology. All clinical isolates were cultured on Sabouraud dextrose agar (Merck, Germany). Candida species were also identified by germ tube formation in serum, chlamydospore pro- duction on corn-meal agar (Difco, USA) and assimilation of carbon sources in the API 20 C AUX yeast identification system (Bio merieux, France).

\subsection{Extraction of Genomic DNA}

Genomic DNA was extracted using the Phenol-chloroform method after disruption of cells by glass beads. To prepare biological samples, five vials each containing 1 $\mathrm{mL}$ of serum from a healthy human donor were spiked respectively with $10^{5}, 10^{4}, 10^{3}, 10^{2}$, and 50 cells of C. albicans, C. parapsilosis, and C. glabrata. Then, DNA was extracted from these samples using the Prime prep Genomic DNA Isolation kit according to the Manufacturer's instructions. The DNA was eluted in $20 \mu \mathrm{L}$ of $\mathrm{ddH}_{2} \mathrm{O}$ and $1 \mu \mathrm{L}$ was used as the template in PCR experiments.

\subsection{Polymerase Chain Reaction Amplification}

Polymerase chain reaction was performed using speciesspecific primer pairs (albicans: F -5'ATGTTATTCAAGTCTTTCGTTACT-3',R-5'-TGACATTAAATCCAGATAATTGA GC-3', glabrata:Gla-F-5'-GATGTTGTCACTAAGACTGTTCA-3'،Gla-R5'AGTAGGAAGACAACTTGGCAAGG-3',parapsilosis:Par-F5'GAGGATGAAGTGGAGTCG-3',Par-R-5'CTTCCGAAGCTAGCCAAGT-3') (4).

The PCR was performed in a $25 \mu \mathrm{L}$ reaction mixture containing $12.5 \mu \mathrm{L}$ of Mastermix (Sina gene), $2 \mu \mathrm{L}$ of each primer $(10 \mathrm{mM}), 1 \mu \mathrm{L}$ of template DNA, and sterile distilled water to bring the total volume to $25 \mu \mathrm{L}$. Polymerase Chain Reaction amplification conditions were five minutes of denaturation at $95^{\circ} \mathrm{C}$, followed by 30 cycles at $95^{\circ} \mathrm{C}$ for one minute, $58^{\circ} \mathrm{C}$ for one minute, and $72^{\circ} \mathrm{C}$ for one minute, with final extension step at $72^{\circ} \mathrm{C}$ for one minute and $35^{\circ} \mathrm{C}$ for one minute. Micro tubes were placed inside Eppendorf Master Cycler Gradient for DNA amplification. Also, appropriate positive and negative controls were included in each run.

Polymerase Chain Reaction products were analyzed by electrophoresis on $2 \%$ agarose gel (200 mA, $140 \mathrm{~V})$, visualized by staining with ethidium bromide, and photographed using the imaging UV transilluminator apparatus. The PCR reaction was performed using three primer pairs and a single Candida species DNA. Also PCR reaction was performed using three different Candida species DNA samples and a single species-specific primer pair. All PCR assays were also performed with DNAs extracted from $C$. tropicalis, C. krusei, Saccharomyces cerevisiae, Cryptococcus spp. and Aspergillus spp. Finally, PCR products were purified with the Silica Bead DNA Gel Extraction Kit (K0513) and were sent to Bioneer Co. for sequencing with the specific primers.

\subsection{Extraction of RNA From Candida albicans, and Real-Time Polymerase Chain Reaction (RT-PCR)}

For total RNA isolation, $300 \mu \mathrm{L}$ lysis buffer and $300 \mu \mathrm{L}$ of acid-washed glass beads and a phenol-chloroform- 
Bineshian F et al.

isoamyl alcohol (25:24:1) mixture were added to the cell pellet and vortexed for 20 minutes at $4{ }^{\circ} \mathrm{C}$ in order to lyse the yeast cells (17). Total RNA concentrations were quantified using a spectrophotometer. Equal amounts of RNA were used for RT-PCR. Equal amounts of $1 \mu \mathrm{g}$ of total RNA from each sample were treated with DNase I (Fermentas), according to the manufacturer's instructions. Then, cDNA was synthesized with the Maxime RT Pre Mix kit (iNt RON), following the manufacturer's instructions. The MP65 primers were designed on the basis of the published sequence of the MP65 gene of C. albicans (NCBI, accession:XM_709288). ACT1 gene (F-5'-GTCACCAACTGGGACGAC-3' and R-5'-CGCTCGTTGCCAATGGTG-3') was used as the endogenous reference gene (15). Polymerase chain reaction (PCR) was performed with the following conditions: an initial cycle with five minutes of denaturation at $95^{\circ} \mathrm{C}$, followed by 35 cycles of denaturation for one minute at $95^{\circ} \mathrm{C}$, annealing for one minute at $57^{\circ} \mathrm{C}$, and extension for one minute at $72^{\circ} \mathrm{C}$, followed by $72^{\circ} \mathrm{C}$ for 10 minutes and $35^{\circ} \mathrm{C}$ for one minute.

\subsection{Quantitative Real-Time-Assay}

Evaluation of C. albicans MP65 gene expression was performed using the quantitative real-time PCR technique. Light Cycler Fast Start DNA Master plus SYBR Green1 (Roche) was used according to the manufacturer's instructions. The amplification program was performed at $95^{\circ} \mathrm{C}$ for 10 minutes as the initial denaturation step followed by a 45 -cycle PCR step including five seconds at $95^{\circ} \mathrm{C}, 10$ seconds at $57^{\circ} \mathrm{C}$ and 15 seconds at $72^{\circ} \mathrm{C}$. A negative control (water as template) was included in each run. Standard curves for each gene were established with five-serially diluted cDNA samples. The 2- $\Delta \Delta$ CT method was used to analyze relative changes in gene expression of MP65 for statistical analysis (18). Non-parametric Wilcoxon test was carried out using the SPSS version 16 software.

\subsection{Systemic Infection of Mice}

Candida albicans cells were counted and suspended to a density of $5 \times 10^{6}$ cells $\mathrm{mL}^{-1}$ in sterile PBS. Meanwhile, $10 \mathrm{Balb} / \mathrm{c}$ female mice (18 - $21 \mathrm{~g}$ ) were intravenously injected $0.1 \mathrm{~mL}$ of this suspension, via the lateral tail vein. Ten mice of each group were observed daily for 30 days to monitor survival. Mice of each group were sacrificed on the first, second, fifth, and $21^{\text {st }}$ days following infection, and the left and right kidneys were removed for Colony Forming Unit (CFU) determination and histopathological observations, respectively. The left kidneys and part of the brain were separately homogenized in $10 \mathrm{~mL}$ of saline and serial dilutions plated on Sabouraud dextrose agar supplemented with chloramphenicol. The plates were incubated at $37^{\circ} \mathrm{C}$ for 48 hours. Colonies were then streaked onto CHROM agar plates to confirm identification. For histological observations, the right kidneys and part of the brain were removed from mice and immedi- ately fixed in 10\% (v/v) neutral buffered formalin. Tissues were then stained with hematoxylin and eosin and Periodic Acid-Schiff (PAS) stains and observed under a light microscope. One milliliter of mice blood was used to extract RNA.

\section{Results}

Thirty-six C. albicans isolates (68\%) and six C. glabrata isolates (12\%) were from 50 vulvovaginal candidiasis patients and 66 C. albicans isolates were from patients with HIV with oral candidiasis and one isolate was from the department of Mycology, Tehran University of Medical Sciences. Candida parapsilosis were identified by microbiological methods, the germ tube test, and API 20C AUX. Sixty-six C. albicans isolates from patients with HIV with oral candidiasis and 34 C. albicans isolates from women with vulvovaginal candidiasis were successfully detected and identified by specific PCR primers directed to the MP65 gene. The PCR was performed using specific primers of $C$. albicans and the PCR products contained the $100 \%$ specific 475 bp band (Figures 1 and 2).

Six C. glabrata isolates and one C. parapsilosis isolate were also identified by the specific PCR primers directed to the MP65 genes with all results being consistent with the culture results. The PCR product was $100 \%$ specific. Candida glabrata with the expected band ( $361 \mathrm{bp}$ ) and C. parapsilosis with the expected band (124 bp) were observed (Figure 1). The results showed that the primers used to detect Candida species were relatively specific. In order to determine the sensitivity of PCR, $1 \mathrm{~mL}$ of serum from a normal healthy donor was spiked respectively with $10^{5}, 10^{4}, 10^{3}, 10^{2}$ and 50 cells of $C$. albicans. The DNAs were extracted from these samples using two methods, the Prime Prep Genomic DNA Isolation kit and the phenol-chloroform method, according to the Manufacturer's instructions. Polymerase Chain Reaction was performed and the minimum amount of detectable genomic DNA with phenol-chloroform was 50 yeast cells. Using the Prime Prep Genomic DNA Isolation kit (from Blood), $10^{5}$ yeast cells could be detected.

Comparison of these two methods indicated that the phenol-chloroform method was more sensitive than the other method with 50 yeast cells. Purified PCR products were sequenced and using the NCBI BLAST Software, the results showed that isolates from patients with HIV infected with oral candidiasis (100\%) and isolates from women with vulvovaginal candidiasis (98\%) were consistent with C. albicans. Also sequences of the other isolates (98\%) were similar to C. glabrata and C. parapsilosis.

\subsection{Real Time-Polymerase Chain Reaction Test}

After synthesis of cDNA, PCR with specific primers MP65 and ACT1 (to ensure equal amounts of RNA in c DNA synthesis) satisfactory results were obtained of transcription of genes (Figure 3). 
Bineshian F et al.

\subsection{Quantitative Real Time-Polymerase Chain Re- action}

Relative expression of Mp65 gene significantly increased by 2.3 folds, after the injection of $C$. albicans into the mice $(\mathrm{P}<0.05)$. ACT1 gene was used as an endogenous reference gene. The specific products of Real-Time PCR were determined by melting curve analysis (Figure 4).

Figure 1. Agarose Gel Electrophoresis of the Polymerase Chain Reaction Products of Different Candida Species Using Species-Specific Primer Pairs

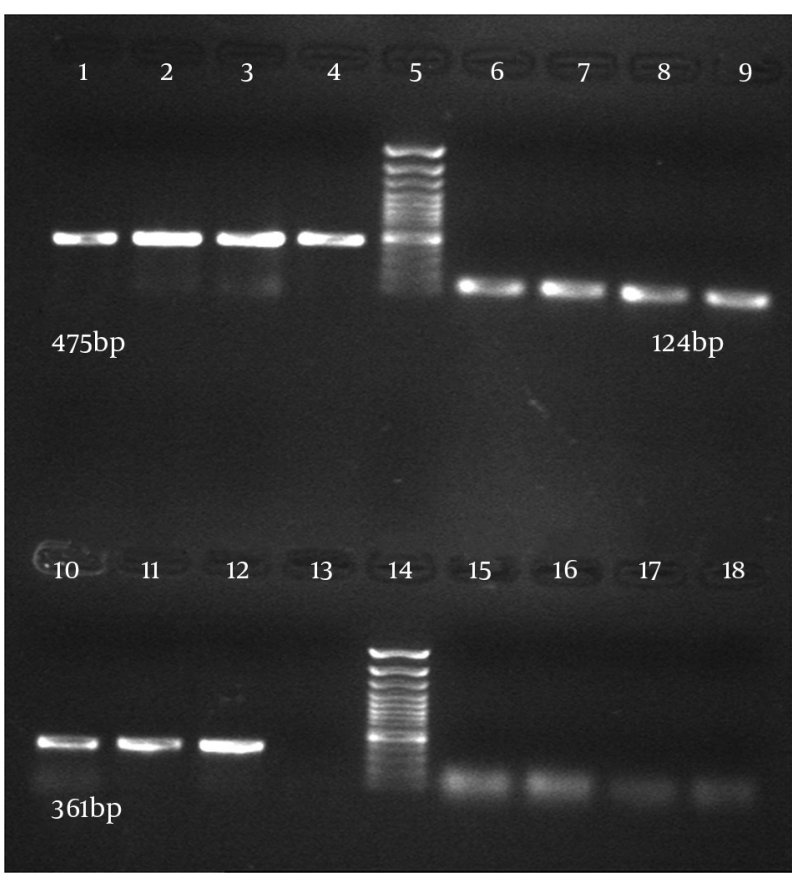

C. albicans (lane 1 - 4), DNA ladder plus $100 \mathrm{bp}$ (lane 5), C. parapsilosis (lane 6-9), C. glabrata (lane 10 -12), $\mathrm{H}_{2} \mathrm{O}$ as negative control (lane 13), DNA ladder plus 100 bp (lane 14), Aspergillus (lane 15), S. cerevisiae (lane 16), C. tropicalis (lane 17), C. krusei (lane 18).
Figure 2. Agarose Gel Electrophoresis of the Polymerase Chain Reaction Products

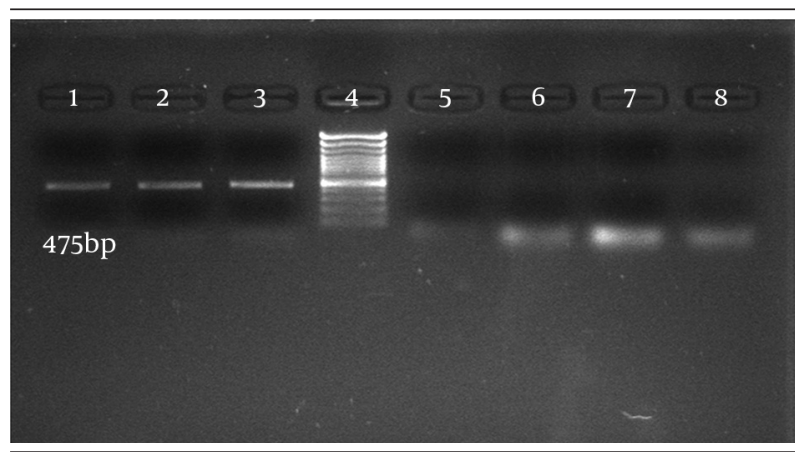

Clinical C. albicans (lane 1 - 3), DNA ladder plus 100 bp (lane 4), Cryptococcus (lane 5), S. cerevisiae (lane 6), C. tropicalis (lane 7), C. krusei (lane 8).

Figure 3. Gel Electrophoresis of Semi-Quantitative Real Time-Polymerase Chain Reaction Products of MP65 Gene from Candida Albicans (Lane1) and ACT1 (Lane 2)

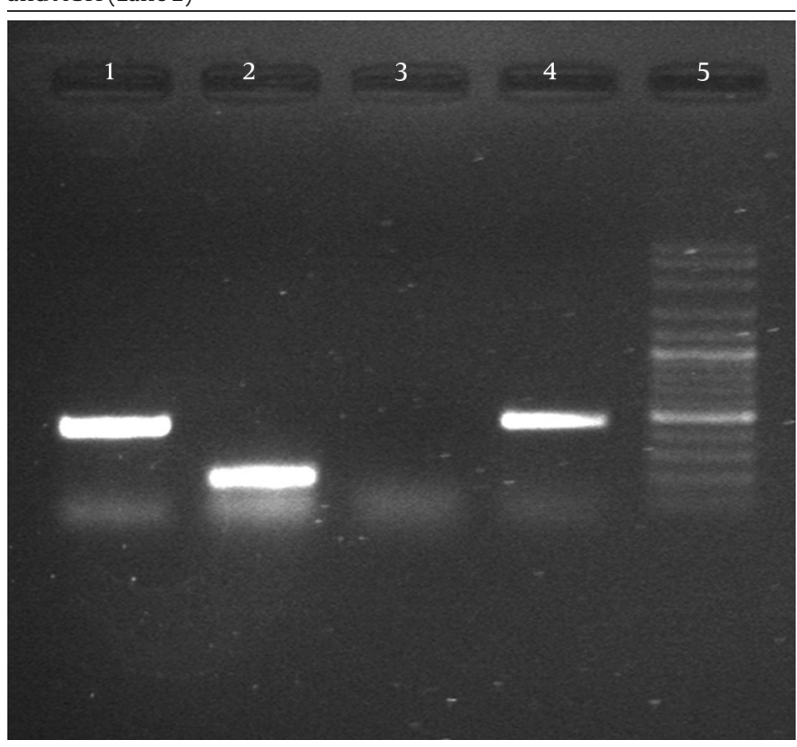

The negative control (lane 3), MP65 gene of C. albicans (ATCC 10231 lane 4) DNA ladder plus $100 \mathrm{bp}$ (lane 5).

Figure 4. Specificity of Light Cycler Polymerase Chain Reaction for Amplification of Candida albicans Gene

A
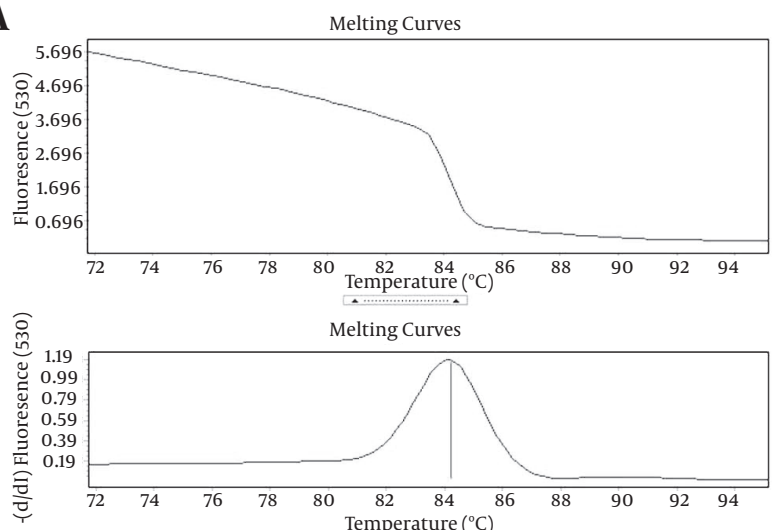

B

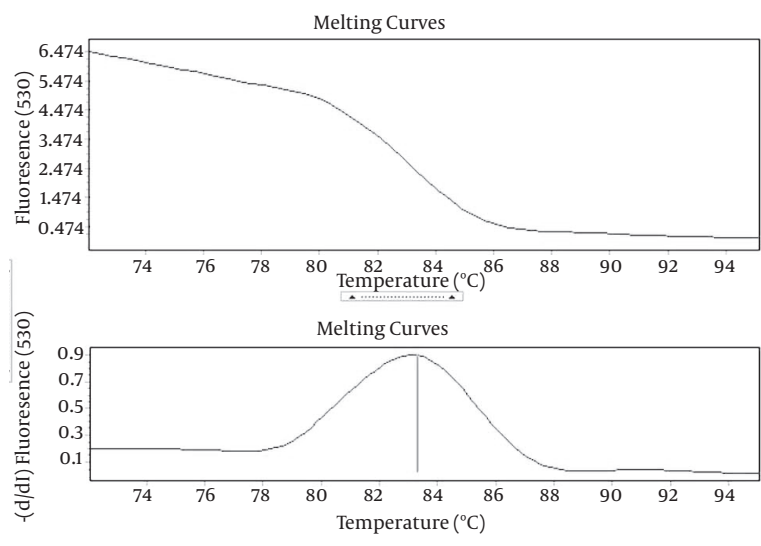

A, MP65; and B, ACT1 determined by melting curve analysis. 
Bineshian F et al.

\section{Discussion}

Increased incidence of invasive candidiasis is directly associated with the increase in immunocompromised patients. Rapid onset of disseminated candidiasis treatment is important in reducing mortality $(1,3,4)$. Candida species cause $11 \%$ of bloodstream infections (19). Therefore, the purpose of molecular-based diagnosis is to detect the infection faster than the conventional methods. Obtaining positive results in patients with candidemia with negative blood culture results are especially important. Susceptibility of Candida species to antifungal drugs may be widely variable. Identification of Candida species is necessary for proper treatment (5). Therefore, the use of PCR for rapid identification of pathogenic opportunistic yeasts, which is a method with high sensitivity and specificity in comparison with serological, biochemical, and microbiological methods, is recommended $(16,19,20)$.

In the current study, PCR assays were applied to detect Candida species using species-specific primer pairs directed to their MP65 gene, encoding mannoproteins. Polymerase Chain Reaction results of all the tested species were consistent (100\% positivity) with culture results and an expected band of $475 \mathrm{bp}, 361 \mathrm{bp}$ and $124 \mathrm{bp}$ for $C$. albicans, C. parapsilosis and C. glabrata, was identified, respectively. The DNAs of all other tested fungal genera $(S$. cerevisiae, Cryptococcus spp. and Aspergillus spp.) were not amplified by any of the Candida species-specific primer pairs. The BLAST software was employed to sequence and analyze all of the PCR products to confirm the results obtained by the PCR method on target gene sequences. The BLAST results showed that isolates of C. albicans, C. glabrata and C. parapsilosis had 100\%, 98\% and 98\%, homology to isolates of XM_709288, CR380959 and HE605208, respectively. The findings of the current study showed that using specific primers to identify C. albicans, C. glabrata and C. parapsilosis species gave outcomes consistent with the results of previous studies from Italy $(4,21)$.

Arancia et al. previously reported that the gene-specific primers of Mp65 of C. albicans in biological samples, by PCR and real-time PCR, were able to detect $C$. albicans in biological samples $(21,22)$. In the present study, Candida species were identified using Mp65 gene-specific primers, and the results were consistent with the mentioned studies indicating that the Mp65 gene is suitable for detection of Candida species. The ability of C. albicans to switch from yeast to hyphal growth is essential for its virulence. Cell wall is a dynamic structure that is reconstructed when fungal cells are subject to hyphal growth, mutations of genes coding for cell wall components (23). Several studies were done in Italy, in which Mp65 of $C$. albicans was a major target of anti-Candida immune responses in humans and mice $(1,8,13,24)$ and another research Mp65 was a putative b-glucanase adhesin which plays a critical role in hyphal formation and virulence of this fungus (14). These data showed that Mp65 plays an important role in membrane or cell wall stability. The
MP65 gene is also required for cell wall integrity and adherence to epithelial cells and biofilm formation in C. albicans (13).

Biofilms are typically found on medical devices such as catheter surfaces and they have attracted the attention of researches because of their persistence and resistance to anti-fungal agents $(25,26)$. De Bernardis et al. previously showed that mp65 $\Delta$ mutant loses adherence to polystyrene plates (27). Heilmann et al. reported that MP65 expression increased at $37^{\circ} \mathrm{C}$ compared to $30^{\circ} \mathrm{C}(10)$. Research has shown that MP65 is one of the several genes affecting the virulence of Candida spp., hyphae formation and adhesion properties. In the current research Mp65 gene expression level of $C$. albicans in mice was higher after injection by 2.3 folds compared to the same before injection. According to the results of the 2- $\Delta \Delta C T$ analysis, relative expression of the Mp65 gene in mice increased significantly after injection compared to pre-injection ( $\mathrm{P}$ $<0.05$ ). Real-time PCR assay data analysis showed that the MP65 gene expression levels in mice after injection was significantly higher than that pre-injection. It seems that even though the MP65 gene is expressed in both yeast and hyphae, when there is yeast in the serum and the temperature is $37^{\circ} \mathrm{C}$ the level of expression increases.

The employed method proved to be sensitive and specific for C. albicans, C. glabrata and C. parapsilosis. The importance of this method was its capability with minimum molecular laboratory facilities. It is important to mention that the Light Cycler technique and high-resolution melting analysis are expensive or require technologies not available in all laboratories $(14,28)$. The method used in this study is rapid, specific and reproducible. Using the PCR method with specific primer pairs to identify Candida species is cost-effective because, no DNA probes or expensive analysis are needed. There is also the possibility of Candida detection directly from serum and blood samples taken from patients with invasive candidiasis. Early initiation of antifungal therapy is crucial to reduce the mortality rate associated with disseminated candidiasis.

\section{Acknowledgements}

The authors wish to thank Dr. Mirhendi, and Dr. M. Geramishaar for providing the Candida parapsilosis isolates.

\section{Authors' Contributions}

All authors of this research paper directly participated in the study.

\section{Funding/Support}

This study was supported by the Tarbiat Modares University of Tehran, Iran. Tarbiat Modares University financially supported this study, and Semnan University of Medical Sciences and Tehran Blood Transfusion Research Center provided the laboratory facilities. 


\section{References}

1. La Valle R, Sandini S, Gomez MJ, Mondello F, Romagnoli G, Nisini $\mathrm{R}$, et al. Generation of a recombinant 65-kilodalton mannoprotein, a major antigen target of cell-mediated immune response to Candida albicans. Infect Immun. 2000;68(12):6777-84.

2. Berzaghi R, Colombo AL, Machado AM, de Camargo ZP. New approach for diagnosis of candidemia based on detection of a 65-kilodalton antigen. Clin Vaccine Immunol. 2009;16(11):1538-45.

3. Gudlaugsson O, Gillespie S, Lee K, Vande Berg J, Hu J, Messer S, et al. Attributable mortality of nosocomial candidemia, revisited. Clin Infect Dis. 2003;37(9):1172-7.

4. Arancia S, Sandini S, Cassone A, De Bernardis F. Use of 65kDa mannoprotein gene primers in PCR methods for the identification of five medically important Candida species. Mol Cell Probes. 2009;23(5):218-26.

5. Pappas PG, Rex JH, Sobel JD, Filler SG, Dismukes WE, Walsh TJ, et al. Guidelines for treatment of candidiasis. Clin Infect Dis. 2004;38(2):161-89.

6. Kim J, Sudbery P. Candida albicans, a major human fungal pathogen. J Microbiol. 2011;49(2):171-7.

7. Sudbery PE. Growth of Candida albicans hyphae. Nat Rev Microbiol. 2011;9(10):737-48.

8. Gomez MJ, Maras B, Barca A, La Valle R, Barra D, Cassone A. Biochemical and immunological characterization of MP65, a major mannoprotein antigen of the opportunistic human pathogen Candida albicans. Infect Immun. 2000;68(2):694-701.

9. Gow NA, van de Veerdonk FL, Brown AJ, Netea MG. Candida albicans morphogenesis and host defence: discriminating invasion from colonization. Nat Rev Microbiol. 2011;10(2):112-22.

10. Heilmann CJ, Sorgo AG, Siliakus AR, Dekker HL, Brul S, de Koster CG, et al. Hyphal induction in the human fungal pathogen Candida albicans reveals a characteristic wall protein profile. Microbiology. 2011;157(Pt 8):2297-307.

11. $\mathrm{Xu} \mathrm{H}$, Nobile CJ, Dongari-Bagtzoglou A. Glucanase induces filamentation of the fungal pathogen Candida albicans. PLoS One. 2013;8(5):e63736.

12. Sorgo AG, Heilmann CJ, Brul S, de Koster CG, Klis FM. Beyond the wall: Candida albicans secret(e)s to survive. FEMS Microbiol Lett. 2013;338(1):10-7.

13. Sandini S, Stringaro A, Arancia S, Colone M, Mondello F, Murtas S, et al. The MP65 gene is required for cell wall integrity, adherence to epithelial cells and biofilm formation in Candida albicans. BMC Microbiol. 2011;11:106.

14. Sandini S, La Valle R, De Bernardis F, Macri C, Cassone A. The 65 $\mathrm{kDa}$ mannoprotein gene of Candida albicans encodes a putative beta-glucanase adhesin required for hyphal morphogenesis and experimental pathogenicity. Cell Microbiol. 2007;9(5):1223-38.

15. Malani PN, Bradley SF, Little RS, Kauffman CA. Trends in species causing fungaemia in a tertiary care medical centre over 12 years.
Mycoses. 2001;44(11-12):446-9.

16. Xiang H, Xiong L, Liu X, Tu Z. Rapid simultaneous detection and identification of six species Candida using polymerase chain reaction and reverse line hybridization assay. J Microbiol Methods. 2007;69(2):282-7.

17. Stehr F, Felk A, Gacser A, Kretschmar M, Mahnss B, Neuber K, et al. Expression analysis of the Candida albicans lipase gene family during experimental infections and in patient samples. FEMS Yeast Res. 2004;4(4-5):401-8.

18. Pfaffl MW. A new mathematical model for relative quantification in real-time RT-PCR. Nucleic Acids Res. 2001;29(9):e45.

19. Szabo EK, MacCallum DM. The contribution of mouse models to our understanding of systemic candidiasis. FEMS Microbiol Lett. 2011;320(1):1-8

20. White PL, Shetty A, Barnes RA. Detection of seven Candida species using the Light-Cycler system. J Med Microbiol. 2003;52(Pt 3):229-38.

21. Arancia S, Sandini S, Cassone A, De Bernardis F, La Valle R. Construction and use of PCR primers from a $65 \mathrm{kDa}$ mannoprotein gene for identification of C. albicans. Mol Cell Probes. 2004;18(3):171-5.

22. Arancia S, Carattoli A, La Valle R, Cassone A, De Bernardis F. Use of $65 \mathrm{kDa}$ mannoprotein gene primers in Real Time PCR identification of Candida albicans in biological samples. Mol Cell Probes. 2006;20(5):263-8.

23. Francois JM. A simple method for quantitative determination of polysaccharides in fungal cell walls. Nat Protoc. 2006;1(6):29953000 .

24. Nisini R, Romagnoli G, Gomez MJ, La Valle R, Torosantucci A, Mariotti $S$, et al. Antigenic properties and processing requirements of 65-kilodalton mannoprotein, a major antigen target of antiCandida human T-cell response, as disclosed by specific human T-cell clones. Infect Immun. 2001;69(6):3728-36.

25. Morgunova E, Saller S, Haase I, Cushman M, Bacher A, Fischer $\mathrm{M}$, et al. Lumazine synthase from Candida albicans as an antifungal target enzyme: structural and biochemical basis for drug design. J Biol Chem. 2007;282(23):17231-41.

26. Hiller E, Heine S, Brunner H, Rupp S. Candida albicans Sun41p, a putative glycosidase, is involved in morphogenesis, cell wall biogenesis, and biofilm formation. Eukaryot Cell. 2007;6(11):2056-65.

27. De Bernardis F, Liu H, O'Mahony R, La Valle R, Bartollino S, Sandini $\mathrm{S}$, et al. Human domain antibodies against virulence traits of Candida albicans inhibit fungus adherence to vaginal epithelium and protect against experimental vaginal candidiasis. I Infect Dis. 2007;195(1):149-57.

28. Arancia S, Sandini S, De Bernardis F, Fortini D. Rapid, simple, and low-cost identification of Candida species using high-resolution melting analysis. Diagn Microbiol Infect Dis. 2011;69(3):283-5. 